\title{
Synthesis and Photoluminescent Properties of 2-(3-Carboxymethylindazol-1-yl)anilines
}

\author{
A. A. Shetnev ${ }^{a, *}$, V. A. Panova ${ }^{a}$, P. M. Kutuzova ${ }^{b}$, M. V. Tarasenko ${ }^{a}$, M. V. Zhmykhova ${ }^{c}$, \\ S. V. Baykov ${ }^{c}$, and S. I. Filimonov ${ }^{d}$ \\ ${ }^{a}$ K.D. Ushinsky Yaroslavl State Pedagogical University, Yaroslavl, 150000 Russia \\ ${ }^{b}$ P.G. Demidov Yaroslavl State University, Yaroslavl, 150003 Russia \\ ${ }^{c}$ Institute of Chemistry, St. Petersburg State University, 198504 Russia \\ ${ }^{d}$ Yaroslavl State Technical University, Yaroslavl, 150023 Russia \\ *e-mail: a.shetnev@yspu.org
}

Received April 15, 2021; revised May 11, 2021; accepted May 14, 2021

\begin{abstract}
A two-stage method for the preparation of 2-(3-carboxymethylindazol-1-yl)anilines using the $\mathrm{N}$-arylation reaction of 3-carboxymethylindazoles with $o$-nitrohaloarenes and subsequent reduction of nitrocontaining intermediates with tin(II) chloride was developed. The experimental results showed that the use of the synthesized compounds as fluorophores in the visible region of the spectrum is promising.
\end{abstract}

Keywords: heterocycles, $N$-arylation, reduction, photoluminescence

DOI: $10.1134 / \mathrm{S} 1070363221060049$

$N$-Substituted indazole derivatives have attracted considerable interest of researchers for a long time. They have been found among the alkaloids of black cumin $[1,2]$. These compounds are used as antiemetics (granisetron, tropisetron) [3], anticancer drugs (niraparib, pazopanib) [4], anti-inflammatory agents (benzadac, benzydamine) $[5,6]$. Biological activity of $N$-substituted indazole derivatives have been reviewed in detail in [7-9].

In addition, a wide range of useful photophysical properties of $N$-substituted indazoles should be noted. They are used in lighting technology, for light-emitting devices production, as well as in study of biochemical processes in living systems using fluorescent trackers [10-13].

Due to great practical importance of $\mathrm{N}$-arylated indazoles, over the past two decades methods for their design have been studied in detail and developed using both base and transition metal catalysis [14-19] $\mathrm{N}$-Arylation reactions of $1 \mathrm{H}$-indazoles can proceed at both nitrogen atoms [14-16]. However, as a rule, the reaction proceeds regioselectively at the $\mathrm{N}^{1}$ atom of the indazole ring [17-19]. At the same time, despite a significant data on this topic, only a single publication is devoted to the reaction of $\mathrm{N}$-arylation of indazole-3-carboxylates [20].

Earlier, we have found unusual regioselectivity in the $\mathrm{N}$-arylation reaction of pyrazole-3-carboxylates when using $O$-nitro-substituted haloarenes [21]. Under the reaction conditions, a predominant nucleophilic substitution at the $\mathrm{N}^{2}$ atom of the pyrazole ring was observed. Subsequent reduction of the nitro derivative with tin(II) chloride and other reagents [22] in all cases gave $N$-hydroxyquinoxalines as the products of reductive cyclization. The obtained compounds showed the properties of potent and selective inhibitors of human monoamine oxidase [23]. Herein, we attempted to aply this approach to the synthesis of fused systems containing indazole and quinoxaline rings.

To accomplish this task, methyl indazole-3-carboxylate was reacted with a number of electron-deficient $O$-nitrohaloarenes in absolute DMF in the presence of anhydrous potassium carbonate as a deprotonating agent (Scheme 1). However, it was found that the $N$-arylation of indazole3 -carboxylates proceeds at the $\mathrm{N}^{1}$ atom of the indazole ring to give the corresponding $N$-aryl derivatives $\mathbf{3 a}-\mathbf{3 h}$ 
Scheme 1.

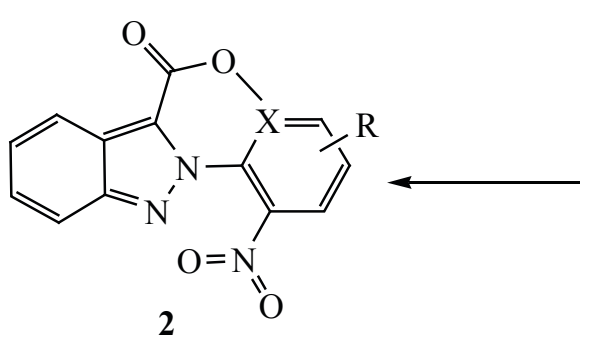

2

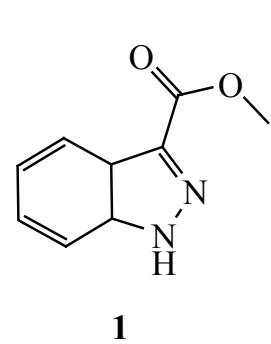

1

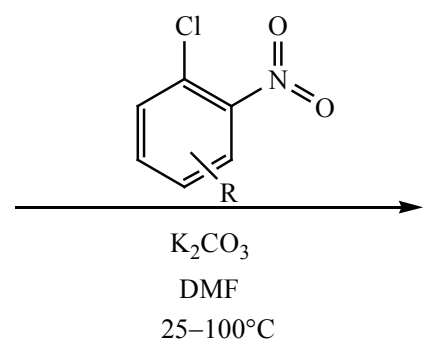

$25-100^{\circ} \mathrm{C}$

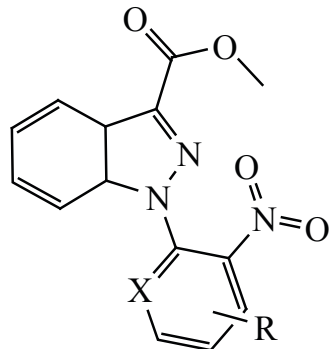

$\mathbf{3 a}-\mathbf{3 h}$

$\mathrm{R}=\mathrm{H}, \mathrm{X}=\mathrm{C}(\mathbf{a}, 93 \%) ; \mathrm{R}=\mathrm{CN}, \mathrm{X}=\mathrm{C}(\mathbf{b}, 93 \%) ; \mathrm{R}=4-\mathrm{NO}_{2}, \mathrm{X}=\mathrm{C}(\mathbf{c}, 97 \%) ; \mathrm{R}=4-\mathrm{COOEt}, \mathrm{X}=\mathrm{C}(\mathbf{d}, 82 \%) ; \mathrm{R}=4-\mathrm{CF} 3$, $\mathrm{X}=\mathrm{C}(\mathbf{e}, 90 \%) ; \mathrm{R}=4-$

Scheme 2.

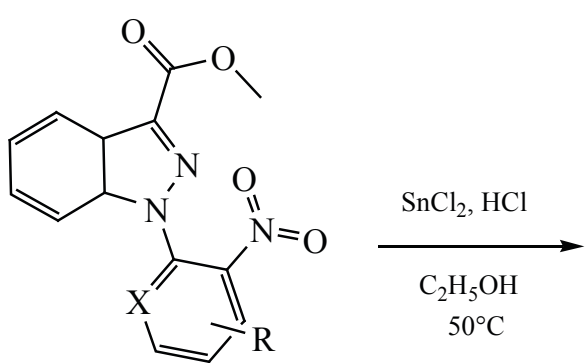

$3 a-3 h$

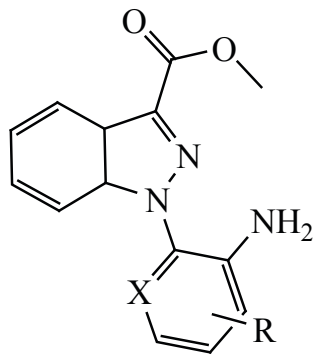

$\mathbf{4 a}-\mathbf{4 h}$

$\mathrm{R}=\mathrm{H}, \mathrm{X}=\mathrm{C}(\mathbf{a}, 84 \%) ; \mathrm{R}=\mathrm{CN}, \mathrm{X}=\mathrm{C}(\mathbf{b}, 59 \%) ; \mathrm{R}=4-\mathrm{NH}_{2}, \mathrm{X}=\mathrm{C}(\mathbf{c}, 61 \%) ; \mathrm{R}=4-\mathrm{COOEt}, \mathrm{X}=\mathrm{C}(\mathbf{d}, 88 \%) ; \mathrm{R}=4-\mathrm{CF}_{3}, \mathrm{X}=\mathrm{C}(\mathbf{e}, 58 \%) ; \mathrm{R}=4-$
$\mathrm{O}$

with yields up to $97 \%$. Despite attempts to vary the reaction conditions (temperature, solvent), the use of deprotonating agents of different nature (potassium tertbutoxide, triethylamine, 4- $N, N$-dimethylaminopyridine) failed to detect the formation of isomeric compounds $\mathbf{2}$.

The obtained series of nitro derivatives $\mathbf{3} \mathbf{a}-\mathbf{3} \mathbf{h}$ was converted into the corresponding amines $\mathbf{4 a - 4 h}$ using the previously developed method for the reduction of $N$-nitroarylpyrazole-3-carboxylates with tin(II) chloride in ethanol in the presence of hydrochloric acid [21] (Scheme 2). Compounds $\mathbf{4 a - 4 h}$ were obtained in yields of $58-88 \%$.
A series of heterocyclic amines $\mathbf{4 a}-\mathbf{4 h}$ containing various functional groups was investigated for the presence of photoluminescence in the solid state at room temperature using a Fluorolog 3 fluorescence spectrometer. The found emission spectral maxima $\left(\lambda_{\mathrm{e} m}^{\max }\right)$, the excited state lifetime $(\tau)$ and the chromaticity diagram parameters (CIE $[x ; y]$ ) are given in Table 1 . The emission spectra recorded upon irradiation with light $\lambda_{\mathrm{em}} 450 \mathrm{~nm}$ are shown in Fig. 1.

The obtained data showed that compounds $\mathbf{4 a}-\mathbf{4 h}$ possess strong yellow-green photoluminescence in the solid state. The emission spectra are mainly represented by a wide band. Luminescence maxima are in the range 
from 487 to $540 \mathrm{~nm}$. The largest shift of the maximum occurs when hydrogen (4a) is replaced by a nitrile group (4e) at position 4 . The lifetimes of the excited state for compounds $\mathbf{4 b}, \mathbf{4 d}-\mathbf{4 h}$ do not differ from each other and are in the range from 0.4 to $4.4 \mathrm{nsec}$. A sharp difference is observed for compounds $\mathbf{4 a}$ and $\mathbf{4} \mathbf{c}$, for which the excited state lifetime is 653 and 518 ns, respectively. Such a sharp increase in the excited state lifetime may be explained by more dense molecular packing due to the strong intermolecular dipole-dipole interaction [24, 25].

In conclusion, a series of new 2-(3-carboxymethylindazol-1-yl)anilines was synthesized by a two-step method, including $\mathrm{N}$-arylation of 3-carboxymethylindazoles with $o$-nitrohaloarenes followed by the reduction of nitro-containing intermediates. The proposed approach provides the overall yield of the target amino derivatives in $62-78 \%$ range. The obtained compounds are of interest as organic fluorophores with emission in the yellow-green spectral region.

\section{EXPERIMENTAL}

Organic, inorganic reagents and solvents were obtained from commercial sources (Aldrich, Vekton, Ekros) and were used without additional purification. The reaction progress was monitored by thin layer chromatography (TLC) on silica gel on Silufol UV aluminum plates eluting with a mixture of ethyl acetate and petroleum ether $(1: 1)$. NMR spectra were recorded on a Varian XL-400 instrument (400 MHz) in DMSO- $d_{6}$ solutions at $25^{\circ} \mathrm{C}$. Melting points were determined on a Büchi M-560 melting point and boiling point apparatus. High-resolution mass spectra were recorded on a Bruker Daltonics MicrOTOF-II instrument, the ionization method was electrospray ionisation (ESI), the temperature of the ionization source was $180^{\circ} \mathrm{C}$, the eluent was methanol. Photoluminescence spectra, excited state lifetimes were obtained on a Fluorolog 3 fluorescence spectrometer (Horiba Jobin Yvon).

General procedure for the synthesis of methyl 1-(2-nitroaryl)-1 $\boldsymbol{H}$-indazole-3-carboxylates $3 \mathrm{a}-\mathbf{3 h}$. To a solution of $1 \mathrm{H}$-indazole-3-carboxylate ( $1 \mathrm{mmol}$ ) in DMF $(1.5 \mathrm{~mL})$ was added the corresponding $O$-nitrohaloarene (1 mmol) and $\mathrm{K}_{2} \mathrm{CO}_{3}(1.3 \mathrm{mmol})$. The reaction mixture was stirred at $70-100^{\circ} \mathrm{C}$ for $12 \mathrm{~h}$. After the reaction completed, the resulting suspension was diluted with $7 \mathrm{~mL}$ of distilled water, the precipitate was filtered off and washed with $2 \mathrm{~mL}$ of water. The resulting product was purified by recrystallization from an ethanol-DMF mixture.
Table 1. Photoluminescent characteristics of compounds $4 a-4 h$

\begin{tabular}{c|l|l|l}
\hline $\begin{array}{c}\text { Comp. } \\
\text { no. }\end{array}$ & $\lambda_{\mathrm{em}}^{\max }, \mathrm{nm}$ & $\tau, \mathrm{ns}$ & CIE $[\mathrm{x} ; \mathrm{y}]$ \\
\hline $\mathbf{4 a}$ & 540 & 653 & $0.40 ; 0.58$ \\
$\mathbf{4 b}$ & 487,520 & 0.4 & $0.35 ; 0.62$ \\
$\mathbf{4 c}$ & 535 & 518 & $0.41 ; 0.56$ \\
$\mathbf{4 s}$ & 515 & 4.4 & $0.37 ; 0.60$ \\
$\mathbf{4 e}$ & 530 & 3.9 & $0.39 ; 0.59$ \\
$\mathbf{4 f}$ & 515 & 4.2 & $0.38 ; 0.60$ \\
$\mathbf{4 g}$ & 512 & 3.3 & $0.35 ; 0.62$ \\
$\mathbf{4 h}$ & 530 & 2.8 & $0.43 ; 0.56$ \\
\hline
\end{tabular}

Methyl 1-(2-nitrophenyl)-1 $\boldsymbol{H}$-indazole-3-carboxylate (3a). Yield $0.276 \mathrm{~g}$ (93\%), pale yellow crystals, mp $163-165^{\circ} \mathrm{C} .{ }^{1} \mathrm{H}$ NMR spectrum, $\delta$, ppm: 3.97 s $\left(3 \mathrm{H}, \mathrm{CH}_{3}\right), 7.49$ d. t $(1 \mathrm{H}, \mathrm{Ar}, J$ 8.4, $2.0 \mathrm{~Hz}), 7.61 \mathrm{~d} . \mathrm{d}(2 \mathrm{H}, \mathrm{Ar}, J 4.0,2.0 \mathrm{~Hz}), 7.87 \mathrm{~d} . \mathrm{t}(1 \mathrm{H}$, $\mathrm{Ar}, J 8.8,2.0 \mathrm{~Hz}), 7.93-8.07 \mathrm{~m}(2 \mathrm{H}, \mathrm{Ar}), 8.22 \mathrm{~d}(1 \mathrm{H}, \mathrm{Ar}$, $J 8.4 \mathrm{~Hz}), 8.27 \mathrm{~d}(1 \mathrm{H}, \mathrm{Ar}, J 8.4 \mathrm{~Hz}) .{ }^{13} \mathrm{C}$ NMR spectrum, $\delta$, ppm: 52.06, 110.43, 121.68, 123.01, 124.26, 125.92, $128.50,128.55,130.64,130.70,134.61,137.18,140.78$, 145.04, 161.81. Mass spectrum, $m / z: 320.0643[M+\mathrm{Na}]^{+}$ (calcd for $\mathrm{C}_{15} \mathrm{H}_{11} \mathrm{~N}_{3} \mathrm{NaO}_{4}$ : 320.0642).

Methyl 1-(4-cyano-2-nitrophenyl)-1 $\mathrm{H}$-indazole3-carboxylate (3b). Yield $0.299 \mathrm{~g}(93 \%)$, pale yellow powder, mp $230-232{ }^{\circ} \mathrm{C} .{ }^{1} \mathrm{H}$ NMR spectrum, $\delta$, ppm: $3.97 \mathrm{~s}\left(3 \mathrm{H}, \mathrm{CH}_{3}\right), 7.53 \mathrm{~s}(1 \mathrm{H}, \mathrm{Ar}), 7.66 \mathrm{~s}(1 \mathrm{H}$, Ar), $7.76 \mathrm{~s}(1 \mathrm{H}, \mathrm{Ar}), 8.23 \mathrm{~d}(1 \mathrm{H}, \mathrm{Ar}, J 8.0 \mathrm{~Hz})$, $8.31 \mathrm{~d}(1 \mathrm{H}, \mathrm{Ar}, J 8.4 \mathrm{~Hz}), 8.49 \mathrm{~d}(1 \mathrm{H}, \mathrm{Ar}, J 8.4 \mathrm{~Hz})$, $8.87 \mathrm{~s}(1 \mathrm{H}, \mathrm{Ar}) .{ }^{13} \mathrm{C}$ NMR spectrum, $\delta_{\mathrm{C}}, \mathrm{ppm}$ :

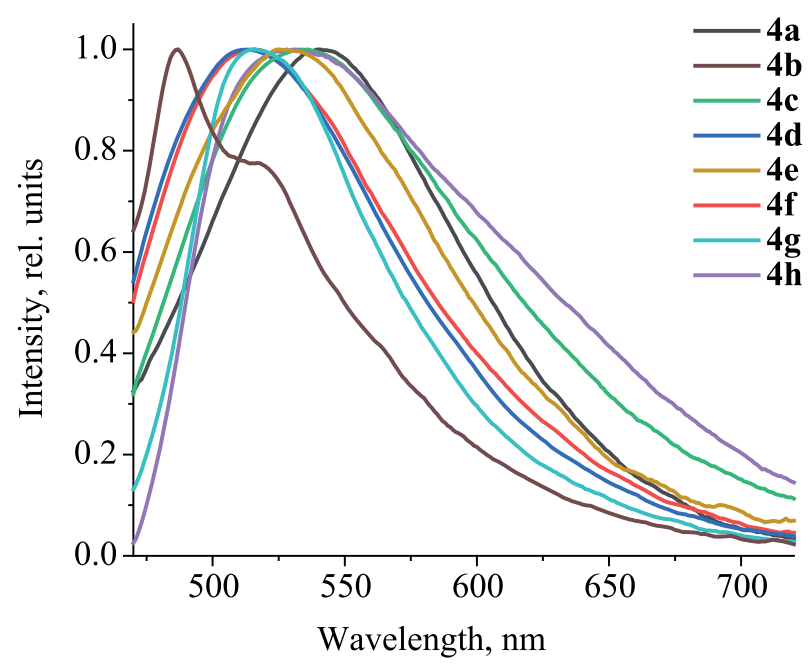

Fig. 1. Photoluminescence spectra of compounds $4 \mathbf{a}-\mathbf{4 h}$. 
52.36, 110.80, 112.61, 116.51, 121.97, 123.31, 124.86, 128.90, 129.07, 130.36, 134.11, 138.24, 138.36, 140.48, 144.66, 161.62. Mass spectrum, $m / z: 345.0596[M+\mathrm{Na}]^{+}$ (calcd for $\mathrm{C}_{16} \mathrm{H}_{10} \mathrm{~N}_{4} \mathrm{NaO}_{4}$ : 345.0594 ).

Methyl 1-(2,4-dinitrophenyl)-1 $\mathrm{H}$-indazole-3-carboxylate (3c). Yield $0.332 \mathrm{~g}(97 \%)$, yellow powder, $\mathrm{mp}$ 186- $189^{\circ} \mathrm{C} .{ }^{1} \mathrm{H}$ NMR spectrum, $\delta$, ppm: $3.98 \mathrm{~s}\left(3 \mathrm{H}, \mathrm{CH}_{3}\right)$, $7.54 \mathrm{t}(2 \mathrm{H}, \mathrm{Ar}, J 7.6 \mathrm{~Hz}), 7.68 \mathrm{t}(1 \mathrm{H}, \mathrm{Ar}, J 7.6 \mathrm{~Hz}), 7.78 \mathrm{~d}$ $(1 \mathrm{H}, \mathrm{Ar}, J 8.0 \mathrm{~Hz}), 8.24 \mathrm{~d}(1 \mathrm{H}, \mathrm{Ar}, J 7.6 \mathrm{~Hz}), 8.37 \mathrm{~d}$ $(1 \mathrm{H}, \mathrm{Ar}, J 8.8 \mathrm{~Hz}), 8.75 \mathrm{~d}(1 \mathrm{H}, \mathrm{Ar}, J 8.4 \mathrm{~Hz}), 9.00 \mathrm{~s}$ $(1 \mathrm{H}, \mathrm{Ar}) .{ }^{13} \mathrm{C}$ NMR spectrum, $\delta_{\mathrm{C}}$, ppm: 52.28, 110.66, $121.80,122.00,123.34,124.85,128.85,129.08,135.22$, 138.57, 140.44, 144.26, 146.65, 161.49. Mass spectrum, $m / z: 365.0493[M+\mathrm{Na}]^{+}$(calcd for $\mathrm{C}_{15} \mathrm{H}_{10} \mathrm{~N}_{4} \mathrm{NaO}_{6}$ : 365.0493).

Methyl 1-[4-(ethoxycarbonyl)-2-nitrophenyl]-1 Hindazole-3-carboxylate (3d). Yield $0.302 \mathrm{~g}(82 \%)$, pale yellow powder, mp $158-161^{\circ} \mathrm{C} .{ }^{1} \mathrm{H}$ NMR spectrum, $\delta$, ppm: $1.39 \mathrm{t}\left(3 \mathrm{H}, \mathrm{CH}_{3}, \mathrm{CH}_{3}, J 7.2 \mathrm{~Hz}\right), 3.97 \mathrm{~s}\left(3 \mathrm{H}, \mathrm{CH}_{3}\right)$, $4.43 \mathrm{q}\left(2 \mathrm{H}, \mathrm{CH}_{2}, J 7.2 \mathrm{~Hz}\right), 7.52 \mathrm{t}(1 \mathrm{H}, \mathrm{Ar}, J 7.6 \mathrm{~Hz}), 7.64 \mathrm{t}$ $(1 \mathrm{H}, \mathrm{Ar}, J 7.6 \mathrm{~Hz}), 7.71 \mathrm{~d}(1 \mathrm{H}, \mathrm{Ar}, J 8.4 \mathrm{~Hz}), 8.22 \mathrm{t}(2 \mathrm{H}, \mathrm{Ar}$, $J 8.4 \mathrm{~Hz}), 8.47 \mathrm{~d}(1 \mathrm{H}, \mathrm{Ar}, J 8.4 \mathrm{~Hz}), 8.65 \mathrm{~s}(1 \mathrm{H}, \mathrm{Ar}) .{ }^{13} \mathrm{C}$ NMR spectrum, $\delta_{\mathrm{C}}$, ppm: 14.01, 52.21, 61.95, 110.59, $121.89,123.23,124.63,126.49,128.66,128.86,131.08$, 134.07, 134.56, 137.99, 140.51, 144.56, 161.65, 163.33 . Mass spectrum, $m / z: 392.0850[M+\mathrm{Na}]^{+}$(calcd for $\mathrm{C}_{18} \mathrm{H}_{15} \mathrm{~N}_{3} \mathrm{NaO}_{6}$ : 392.0853).

Methyl 1-[2-nitrophenyl-4-(trifluoromethyl)]-1Hindazole-3-carboxylate (3e). Yield $0.328 \mathrm{~g}(90 \%)$, beige crystals, mp $182-185^{\circ} \mathrm{C} .{ }^{1} \mathrm{H}$ NMR spectrum, $\delta$, ppm: $3.98 \mathrm{~s}\left(3 \mathrm{H}, \mathrm{CH}_{3}\right), 7.53 \mathrm{t}(1 \mathrm{H}, \mathrm{Ar}, J 7.6 \mathrm{~Hz}), 7.65 \mathrm{t}(1 \mathrm{H}$, Ar, J 7.6 Hz), $7.76 \mathrm{~d}(1 \mathrm{H}, \mathrm{Ar}, J 8.0 \mathrm{~Hz}), 8.24 \mathrm{~d}(1 \mathrm{H}, \mathrm{Ar}$, $J 8.0 \mathrm{~Hz}), 8.32 \mathrm{~d}(1 \mathrm{H}, \mathrm{Ar}, J 8.4 \mathrm{~Hz}), 8.39 \mathrm{~d}(1 \mathrm{H}, \mathrm{Ar}, J$ $8.4 \mathrm{~Hz}), 8.68 \mathrm{~s}(1 \mathrm{H}, \mathrm{Ar}) .{ }^{13} \mathrm{CNMR}$ spectrum, $\delta_{\mathrm{C}}, \mathrm{ppm}: 52.92$, 111.33, 122.00, 122.58, 123.92, 125.36, 129.58, 130.82, 131.93, 134.55, 138.83, 145.59, 162.31. Mass spectrum, $m / z$ : $388.0516[M+\mathrm{Na}]^{+}$(calcd for $\mathrm{C}_{16} \mathrm{H}_{10} \mathrm{~F}_{3} \mathrm{~N}_{3} \mathrm{NaO}_{4}$ : 388.0516).

Methyl 1-[4-(morpholin-4-ylsulfonyl)-2-nitrophenyl]-1 $H$-indazole-3-carboxylate (3f). Yield $0.343 \mathrm{~g}$ (77\%), yellow powder, $\mathrm{mp} 226-230^{\circ} \mathrm{C} .{ }^{1} \mathrm{H} N M R$ spectrum, $\delta$, ppm: $3.10 \mathrm{~s}\left(4 \mathrm{H}, \mathrm{CH}_{2}\right), 3.69 \mathrm{~s}\left(4 \mathrm{H}, \mathrm{CH}_{2}\right), 3.98 \mathrm{~s}(3 \mathrm{H}$, $\left.\mathrm{CH}_{3}\right), 7.54 \mathrm{t}(1 \mathrm{H}, \mathrm{Ar}, J 7.6 \mathrm{~Hz}), 7.66 \mathrm{t}(1 \mathrm{H}, \mathrm{Ar}, J 7.6 \mathrm{~Hz})$, $7.81 \mathrm{~d}(1 \mathrm{H}, \mathrm{Ar}, J 8.4 \mathrm{~Hz}), 8.18-8.34 \mathrm{~m}(3 \mathrm{H}, \mathrm{Ar}), 8.49 \mathrm{~s}(1 \mathrm{H}$, Ar) ${ }^{13} \mathrm{C}$ NMR spectrum, $\delta_{\mathrm{C}}$, ppm: 52.29, 110.86, 121.92, 123.26, 124.76, 125.33, 128.87, 129.48, 133.10, 134.25, 135.97, 138.22, 140.57, 144.72, 161.64. Mass spectrum, $m / z: 469.0787[M+\mathrm{Na}]^{+}$(calcd for $\mathrm{C}_{19} \mathrm{H}_{18} \mathrm{~N}_{4} \mathrm{NaO}_{7} \mathrm{~S}$ : 469.0788).

Methyl 1-(3-nitropyridin-2-yl)-1 H-indazole-3carboxylate (3g). Yield $0.229 \mathrm{~g}(77 \%)$, pale yellow crystals, mp $143-146^{\circ} \mathrm{C} .{ }^{1} \mathrm{H}$ NMR spectrum, $\delta$, ppm: $3.97 \mathrm{~s}\left(3 \mathrm{H}, \mathrm{CH}_{3}\right), 7.55 \mathrm{t}(1 \mathrm{H}, \mathrm{Ar}, J 7.6 \mathrm{~Hz}), 7.70 \mathrm{t}(1 \mathrm{H}$, Ar, J 7.6 Hz), 7.78-7.89 m (1H, Ar), $8.23 \mathrm{~d}(1 \mathrm{H}, \mathrm{Ar}$, $J 8.0 \mathrm{~Hz}), 8.27 \mathrm{~d}(1 \mathrm{H}, \mathrm{Ar}, J 8.4 \mathrm{~Hz}), 8.68 \mathrm{~d}(1 \mathrm{H}, \mathrm{Ar}, J$ $8.0 \mathrm{~Hz}), 8.93 \mathrm{~d}(1 \mathrm{H}, \mathrm{Ar}, J 4.4 \mathrm{~Hz}) .{ }^{13} \mathrm{C}$ NMR spectrum, $\delta_{\mathrm{C}}$, ppm: $52,36,113.32,121.76,123.52,124.13,125.13$, 129.14, 135.57, 138.41, 139.51, 139.72, 142.64, 151.82, 161.46. Mass spectrum, $m / z: 321.0598[M+\mathrm{Na}]^{+}($calcd for $\left.\mathrm{C}_{14} \mathrm{H}_{10} \mathrm{~N}_{4} \mathrm{NaO}_{4}: 321.0594\right)$.

Methyl 1-(5-bromo-3-nitropyridin-2-yl)-1H-indazole-3-carboxylate (3h). Yield $0.312 \mathrm{~g}(83 \%)$, orange crystals, mp $159-162^{\circ} \mathrm{C} .{ }^{1} \mathrm{H}$ NMR spectrum, $\delta$, ppm: $3.96 \mathrm{~s}$ $\left(3 \mathrm{H}, \mathrm{CH}_{3}\right), 7.54 \mathrm{t}(1 \mathrm{H}, \mathrm{Ar}, J 7.2 \mathrm{~Hz}), 7.69 \mathrm{t}(1 \mathrm{H}, \mathrm{Ar}, J$ $8.0 \mathrm{~Hz}), 8.21 \mathrm{~d}(1 \mathrm{H}, \mathrm{Ar}, J 8.4 \mathrm{~Hz}), 8.25 \mathrm{~d}(1 \mathrm{H}, \mathrm{Ar}, J$ $8.8 \mathrm{~Hz}), 9.02 \mathrm{~s}(1 \mathrm{H}, \mathrm{Ar}), 9.07 \mathrm{~s}(1 \mathrm{H}, \mathrm{Ar}) .{ }^{13} \mathrm{C} \mathrm{NMR}$ spectrum, $\delta_{\mathrm{C}}$, ppm: $52.37,117.90,121.80,123.53$, $125.25,137.53,138.74,139.08,139.64,141.44,161.32$. Mass spectrum, $m / z$ : $398.9705[M+\mathrm{Na}]^{+}$(calcd for $\mathrm{C}_{14} \mathrm{H}_{9} \mathrm{BrN}_{4} \mathrm{NaO}_{4}$ : 398.9699).

General procedure for the synthesis of methyl 1-(2-aminoaryl)-1H-indazole-3-carboxylates $4 \mathrm{a}-\mathbf{4 h}$. To a mixture of $\mathrm{SnCl}_{2}(3.5 \mathrm{mmol})$ in conc. $\mathrm{HCl}$ $(2 \mathrm{~mL})$ and ethanol $(2 \mathrm{~mL})$ was added the corresponding 1-(2-nitroaryl)-1 $H$-indazole-3-carboxylate $3(0.01 \mathrm{~mol})$. The reaction mixture was stirred at $50^{\circ} \mathrm{C}$ for $3-4 \mathrm{~h}$. The precipitate was filtered off, washed with ethanol, and recrystallized from an ethanol-DMF mixture.

Methyl 1-(2-aminophenyl)-1H-indazole-3-carboxylate (4a). Yield $0.225 \mathrm{~g}(84 \%)$, pale yellow powder, mp $128-130^{\circ} \mathrm{C} .{ }^{1} \mathrm{H}$ NMR spectrum, $\delta$, ppm: $3.96 \mathrm{~s}(3 \mathrm{H}$, $\left.\mathrm{CH}_{3}\right), 5.13 \mathrm{~s}\left(2 \mathrm{H}, \mathrm{NH}_{2}\right), 6.72 \mathrm{t}(1 \mathrm{H}, \mathrm{Ar}, J 7.2 \mathrm{~Hz}), 6.96 \mathrm{~d}$ $(1 \mathrm{H}, \mathrm{Ar}, J 8.0 \mathrm{~Hz}), 7.20 \mathrm{~d}(1 \mathrm{H}, \mathrm{Ar}, J 7.6 \mathrm{~Hz}), 7.26 \mathrm{t}(1 \mathrm{H}$, Ar, $J 7.6 \mathrm{~Hz}), 7.34 \mathrm{~d}(1 \mathrm{H}, \mathrm{Ar}, J 8.4 \mathrm{~Hz}), 7.41 \mathrm{t}(1 \mathrm{H}, \mathrm{Ar}$, $J 7.2 \mathrm{~Hz}), 7.50 \mathrm{t}(1 \mathrm{H}, \mathrm{Ar}, J 7.6 \mathrm{~Hz}), 8.18 \mathrm{~d}(1 \mathrm{H}, \mathrm{Ar}, J$ $8.0 \mathrm{~Hz}) .{ }^{13} \mathrm{C}$ NMR spectrum, $\delta_{\mathrm{C}}$, ppm: $51.75,111.15$, 116.73, 116.93, 121.35, 123.04, 123.48, 127.39, 130.05, 135.76, 140.91, 143.36, 162.32. Mass spectrum, $m / z$ : $290.0908[M+\mathrm{Na}]^{+}$(calcd for $\mathrm{C}_{15} \mathrm{H}_{13} \mathrm{~N}_{3} \mathrm{NaO}_{2}: 290.0900$ ).

Methyl 1-(2-amino-4-cyanophenyl)-1H-indazole3-carboxylate (4b). Yield $0.169 \mathrm{~g}$ (59\%), pale yellow powder, mp $176-182^{\circ} \mathrm{C} .{ }^{1} \mathrm{H}$ NMR spectrum, $\delta$, ppm: $3.96 \mathrm{~s}$ $\left(3 \mathrm{H}, \mathrm{CH}_{3}\right), 5.69 \mathrm{~s}\left(1 \mathrm{H}, \mathrm{NH}_{2}\right), 7.10 \mathrm{~d}(1 \mathrm{H}, \mathrm{Ar}, J 8.0 \mathrm{~Hz})$, $7.31 \mathrm{~s}(1 \mathrm{H}, \mathrm{Ar}), 7.38-7.44 \mathrm{~m}(3 \mathrm{H}, \mathrm{Ar}), 7.53 \mathrm{t}(1 \mathrm{H}, \mathrm{Ar}, J$ $7.6 \mathrm{~Hz}), 8.19 \mathrm{~d}(1 \mathrm{H}, \mathrm{Ar}, J 8.0 \mathrm{~Hz}) .{ }^{13} \mathrm{C}$ NMR spectrum, 
$\delta_{\mathrm{C}}, \mathrm{ppm}: 51.91,111.14,112.44,118.67,118.83,119.27$, $121.52,123.18,123.77,125.89,127.78,128.86,136.55$, 140.73, 145.07, 162.20. Mass spectrum, $\mathrm{m} / \mathrm{z}: 315.0856$ $[M+\mathrm{Na}]^{+}$(calcd for $\mathrm{C}_{16} \mathrm{H}_{12} \mathrm{~N}_{4} \mathrm{NaO}_{2}: 315.0852$ ).

Methyl 1-(2,4-diaminophenyl)-1H-indazole-3carboxylate (4c). Yield $0.173 \mathrm{~g}(61 \%)$, white powder, $\mathrm{mp} 176-180^{\circ} \mathrm{C} .{ }^{1} \mathrm{H}$ NMR spectrum, $\delta$, ppm: $3.95 \mathrm{~s}(3 \mathrm{H}$, $\left.\mathrm{CH}_{3}\right), 4.67 \mathrm{~s}\left(2 \mathrm{H}, \mathrm{NH}_{2}\right), 5.20 \mathrm{~s}\left(2 \mathrm{H}, \mathrm{NH}_{2}\right), 5.98 \mathrm{~d}(1 \mathrm{H}$, $\mathrm{Ar}, J 8.4 \mathrm{~Hz}), 6.11 \mathrm{~s}(1 \mathrm{H}, \mathrm{Ar}), 6.83 \mathrm{~d}(1 \mathrm{H}, \mathrm{Ar}, J 8.0 \mathrm{~Hz})$, $7.31 \mathrm{~d}(1 \mathrm{H}, \mathrm{Ar}, J 8.4 \mathrm{~Hz}), 7.34-7.41 \mathrm{~m}(1 \mathrm{H}, \mathrm{Ar}), 7.46 \mathrm{~T}$ $(1 \mathrm{H}, \mathrm{Ar}, J 7.6 \mathrm{~Hz}), 8.14 \mathrm{~d}(1 \mathrm{H}, \mathrm{Ar}, J 8.0 \mathrm{~Hz}) .{ }^{13} \mathrm{C} \mathrm{NMR}$ spectrum, $\delta_{\mathrm{C}}$, ppm: 51.63, 100.61, 101.02, 104.03, 111.24, $113.73,121.16,122.87,123.22,127.01,127.98,141.29$, 144.76, 148.53, 162.42. Mass spectrum, $\mathrm{m} / \mathrm{z}: 305.1014$ $[M+\mathrm{Na}]^{+}$(calcd for $\mathrm{C}_{15} \mathrm{H}_{14} \mathrm{~N}_{4} \mathrm{NaO}_{2}: 305.1009$ ).

Methyl 1-[2-amino-4-(ethoxycarbonyl)phenyl]-1 $H$ indazole-3-carboxylate (4d). Yield $0.299 \mathrm{~g}(88 \%)$, beige powder, mp $130-135^{\circ} \mathrm{C} .{ }^{1} \mathrm{H}$ NMR spectrum, $\delta$, ppm: $1.35 \mathrm{t}$ $\left(3 \mathrm{H}, \mathrm{CH}_{3}, J 6.8 \mathrm{~Hz}\right), 3.97 \mathrm{~s}\left(3 \mathrm{H}, \mathrm{CH}_{3}\right), 4.34 \mathrm{q}\left(2 \mathrm{H}, \mathrm{CH}_{2}\right.$, $J 7.2 \mathrm{~Hz}$,), $5.49 \mathrm{~s}\left(2 \mathrm{H}, \mathrm{NH}_{2}\right), 7.28 \mathrm{~d}(1 \mathrm{H}, \mathrm{Ar}, J 8.0 \mathrm{~Hz})$, $7.33-7.46 \mathrm{~m}(3 \mathrm{H}, \mathrm{Ar}), 7.53 \mathrm{t}(1 \mathrm{H}, \mathrm{Ar}, J 7.6 \mathrm{~Hz}), 7.61 \mathrm{~s}$ $(1 \mathrm{H}, \mathrm{Ar}), 8.20 \mathrm{~d}(1 \mathrm{H}, \mathrm{Ar}, J 8.0 \mathrm{~Hz}) .{ }^{13} \mathrm{C}$ NMR spectrum, $\delta_{\mathrm{C}}, \mathrm{ppm}: 166.17,162.94,145.01,141.44,136.95,131.87$, $128.40,128.35,126.60,124.37,123.84,122.17,117.84$, 117.06, 111.94, 61.49, 52.54, 14.87. Mass spectrum, $m / z$ : $362.1151[M+\mathrm{H}]^{+}$(calcd for $\mathrm{C}_{18} \mathrm{H}_{17} \mathrm{~N}_{3} \mathrm{NaO}_{4}: 362.1111$ ).

Methyl 1-[2-amino-4-(trifluoromethyl)phenyl]-1 $\mathrm{H}$ indazole-3-carboxylate (4e). Yield $0.195 \mathrm{~g}(58 \%)$, pale yellow powder, mp $154-157^{\circ} \mathrm{C} .{ }^{1} \mathrm{H}$ NMR spectrum, $\delta$, ppm: $3.97 \mathrm{~s}\left(3 \mathrm{H}, \mathrm{CH}_{3}\right), 4.92 \mathrm{~s}\left(2 \mathrm{H}, \mathrm{NH}_{2}\right), 6.99 \mathrm{~d}(1 \mathrm{H}$, Ar, J $8.4 \mathrm{~Hz}), 7.29 \mathrm{~s}(1 \mathrm{H}, \mathrm{Ar}), 7.38-7.45 \mathrm{~m}(3 \mathrm{H}, \mathrm{Ar})$, $7.52 \mathrm{t}(1 \mathrm{H}, \mathrm{Ar}, J 7.6 \mathrm{~Hz}), 8.20 \mathrm{~d}(1 \mathrm{H}, \mathrm{Ar}, J 8.0 \mathrm{~Hz}) .{ }^{13} \mathrm{C}$ NMR spectrum, $\delta_{\mathrm{C}}$, ppm: 52.53, 111.82, 112.48, 113.32, $122.17,123.85,124.37,125.86,126.09,128.38,129.35$, 130.98, 131.29, 141.52, 145.68, 162.93. Mass spectrum, $m / z: 358.0775[M+\mathrm{Na}]^{+}$(calcd for $\mathrm{C}_{16} \mathrm{H}_{12} \mathrm{~F}_{3} \mathrm{~N}_{3} \mathrm{NaO}_{2}$ : 358.0774).

Methyl 1-[2-amino-4-(morpholin-4-ylsulfonyl)phenyl]-1 $\boldsymbol{H}$-indazole-3-carboxylate (4f). Yield $0.337 \mathrm{~g}$ (81\%), pale yellow crystals, mp $227-231{ }^{\circ} \mathrm{C} .{ }^{1} \mathrm{H}$ NMR spectrum, $\delta$, ppm: $2.98 \mathrm{~s}(4 \mathrm{H}, \mathrm{Alk}), 3.68 \mathrm{~s}(4 \mathrm{H}, \mathrm{Alk}), 3.98 \mathrm{~s}$ $\left(3 \mathrm{H}, \mathrm{CH}_{3}\right), 5.75 \mathrm{~s}\left(2 \mathrm{H}, \mathrm{NH}_{2}\right), 7.00 \mathrm{~d}(1 \mathrm{H}, \mathrm{Ar}, J 8.0 \mathrm{~Hz})$, $7.35 \mathrm{~s}(1 \mathrm{H}, \mathrm{Ar}), 7.41-7.57 \mathrm{~m}(4 \mathrm{H}, \mathrm{Ar}), 8.21 \mathrm{~d}(1 \mathrm{H}, \mathrm{Ar}, J$ $7.6 \mathrm{~Hz}) .{ }^{13} \mathrm{C} \mathrm{NMR}$ spectrum, $\delta_{\mathrm{C}}$, ppm: 45.93, 51.86, 65.35, 111.25, 114.21, 115.00, 121.47, 123.15, 123.73, 125.65, 127.70, 128.52, 135.70, 136.47, 140.78, 145.02, 162.21. Mass spectrum, $m / z$ : $439.1053[M+\mathrm{Na}]^{+}($calcd forC ${ }_{19} \mathrm{H}_{20} \mathrm{~N}_{4} \mathrm{NaO}_{5} \mathrm{~S}$ : 439.1047).
Methyl 1-(3-aminopyridin-2-yl)-1H-indazole-3carboxylate (4g). Yield $0.183 \mathrm{~g}(68 \%)$, beige powder, $\mathrm{mp} 145-149^{\circ} \mathrm{C} .{ }^{1} \mathrm{H}$ NMR spectrum, $\delta$, ppm: $3.99 \mathrm{~s}(3 \mathrm{H}$, $\left.\mathrm{CH}_{3}\right), 5.96 \mathrm{~s}\left(2 \mathrm{H}, \mathrm{NH}_{2}\right), 7.20-7.31 \mathrm{~m}(1 \mathrm{H}, \mathrm{Ar}), 7.40 \mathrm{~d}$ $(1 \mathrm{H}, \mathrm{Ar}, J 8.0 \mathrm{~Hz}), 7.46 \mathrm{t}(1 \mathrm{H}, \mathrm{Ar}, J 7.2 \mathrm{~Hz}), 7.57 \mathrm{t}(1 \mathrm{H}$, Ar, $J 7.6 \mathrm{~Hz}), 7.86 \mathrm{~d} . \mathrm{d}(1 \mathrm{H}, \mathrm{Ar}, J 4.4,2.0 \mathrm{~Hz}), 8.10 \mathrm{~d}$ $(1 \mathrm{H}, \mathrm{Ar}, J 8.4 \mathrm{~Hz}), 8.21 \mathrm{~d}(1 \mathrm{H}, \mathrm{Ar}, J 8.0 \mathrm{~Hz}) .{ }^{13} \mathrm{C} \mathrm{NMR}$ spectrum, $\delta_{\mathrm{C}}$, ppm: 51.97, 113.86, 121.19, 122.80, 124.17, 124.24, 124.74, 127.81, 135.16, 136.21, 137.91, 140.29, 162.01. Mass spectrum, $m / z: 291.0854[M+\mathrm{Na}]^{+}($calcd for $\mathrm{C}_{14} \mathrm{H}_{12} \mathrm{~N}_{4} \mathrm{NaO}_{2}$ : 291.0852).

Methyl 1-(3-amino-5-bromopyridin-2-yl)-1Hindazole-3-carboxylate (4h). Yield $0.264 \mathrm{~g}$ (76\%), yellow powder, mp $176-180^{\circ} \mathrm{C} .{ }^{1} \mathrm{H}$ NMR spectrum, $\delta$, ppm: $3.99 \mathrm{~s}\left(3 \mathrm{H}, \mathrm{CH}_{3}\right), 6.23 \mathrm{~s}\left(2 \mathrm{H}, \mathrm{NH}_{2}\right), 7.47 \mathrm{t}(1 \mathrm{H}, \mathrm{Ar}$, $J 7.6 \mathrm{~Hz}), 7.54-7.65 \mathrm{~m}(2 \mathrm{H}, \mathrm{Ar}), 7.92 \mathrm{~s}(1 \mathrm{H}, \mathrm{Ar}), 8.09 \mathrm{~d}$ $(1 \mathrm{H}, \mathrm{Ar}, J 8.0 \mathrm{~Hz}), 8.21 \mathrm{~d}(1 \mathrm{H}, \mathrm{Ar}, J 7.6 \mathrm{~Hz}) .{ }^{13} \mathrm{C} \mathrm{NMR}$ spectrum, $\delta_{\mathrm{C}}$, ppm: 52.01, 113.76, 119.06, 121.26, 122.80, $124.32,125.85,128.00,134.74,135.04,135.55,139.34$, $140.23,161.87$. Mass spectrum, $m / z: 368.9960[M+\mathrm{Na}]^{+}$ (calcd for $\mathrm{C}_{14} \mathrm{H}_{11} \mathrm{BrN}_{4} \mathrm{NaO}_{2}$ : 368.9958).

\section{AUTHOR INFORMATION}

A.A. Shetnev, ORCID: http://orcid.org/0000-0002-4389$461 \mathrm{X}$

V.A. Panova, ORCID: http://orcid.org/0000-0002-47755326

M.V. Tarasenko, ORCID: http://orcid.org/0000-0001-57202664

M.V. Zhmykhova, ORCID: http://orcid.org/0000-00019716-1203

S.V. Baykov, ORCID: http://orcid.org/0000-0002-89125816

S.I. Filimonov, ORCID: http://orcid.org/0000-0001-99034099

\section{FUNDING}

This work was financially supported by the Russian Science Foundation (project no. 20-73-00203) using the equipment of the Resource Centers "Chemical Analysis and Materials Research Center" and "Center for Optical and Laser Materials Research" of St. Petersburg State University.

\section{CONFLICT OF INTEREST}

No conflict of interest was declared by the authors. 


\section{OPEN ACCESS}

This article is licensed under a Creative Commons Attribution 4.0 International License, which permits use, sharing, adaptation, distribution and reproduction in any medium or format, as long as you give appropriate credit to the original author(s) and the source, provide a link to the Creative Commons license, and indicate if changes were made. The images or other third party material in this article are included in the article's Creative Commons license, unless indicated otherwise in a credit line to the material. If material is not included in the article's Creative Commons license and your intended use is not permitted by statutory regulation or exceeds the permitted use, you will need to obtain permission directly from the copyright holder. To view a copy of this license, visit http://creativecommons.org/licenses/by/4.0/.

\section{REFERENCES}

1. Liu, Y.M., Yang, J.S., and Liu, Q.H., Chem. Pharm. Bull., 2004, vol. 52, p. 454.

https://doi.org/10.1248/cpb.52.454

2. Atta-ur-Rahman Malik, S., Hasan, S.S., Choudhary, M.I., Ni, C.-Z., and Clardy, J., Tetrahedron Lett., 1995, vol. 36, p. 1993. https://doi.org/10.1016/0040-4039(95)00210-4

3. Fischer, J. and Ganellin, C.R., John Wiley \& Sons, 2006, p. 448.

https://doi.org/10.1002/3527608001

4. Scott, L.J., Drugs, 2017, vol. 77, p. 1029. https://doi.org/10.1007/s40265-017-0752-y

5. Shen, T.Y., Ann. Rep. Med. Chem., 1968, vol. 3, p. 215. https://doi.org/10.1016/S0065-7743(08)61326-3

6. Gaikwad, D.D., Chapolikar, A.D., Devkate, C.G., Warad, K.D., Tayade, A.P., Pawar, R.P., and Domb, A.J., Eur. J. Med. Chem., 2015, vol. 90, p. 707. https://doi.org/10.1016/j.ejmech.2014.11.029

7. Dong, J., Zhang, Q., Wang, Z., Huang, G., and Li, S., ChemMedChem, 2018, vol. 13, no. 15, p. 1490. https://doi.org/10.1002/cmdc.201800253

8. Wan, Y.C., He, S.Z., Li, W., and Tang, Z.L., Anti-Cancer Agents Med. Chem., 2018, vol. 18, p. 1228.

9. Zhang, S.G., Liang, C.G., and Zhang, W.H., Molecules, 2018, vol. 23, no. 11, p. 2783. https://doi.org/10.3390/molecules23112783

10. Kim, O.S., Jang, J.H., Kim, H.T., Han, S.J., Tsui, G.C., and Joo, J.M., Org. Lett., 2017, vol. 19, no. 6, p. 1450. https://doi.org/10.1021/acs.orglett.7b00410

11. Janardhanan, J.C., Mishra, R.K., Das, G., Sini, S., Jayamurthy, P., Suresh, C.H., Praveen, V.K., Manoj, N., and Babu, B.P., Asian J. Org. Chem., 2018, vol. 7, p. 2094. https://doi.org/10.1002/ajoc.201800413
12. Pakjoo, V., Roshani, M., Pordel, M., and Hoseini, T., Arkivoc, 2012, vol. 9, p. 195. https://doi.org/10.3998/ark.5550190.0013.917

13. Cheng, Y., Li, G., Liu, Y., Shi, Y., Gao, G., Wu, D., Lan, J., and You, J., J. Am. Chem. Soc., 2016, vol. 138, no. 14, p. 4730. https://doi.org/10.1021/jacs.5b09241

14. Ding, X., Bai, J., Wang, H., Zhao, B., Li, J., and Ren, F., Tetrahedron, 2017, vol. 73, p. 172. https://doi.org/10.1016/j.tet.2016.11.066

15. Zhang, R., Liu, Z., Peng, Q., Zhou, Y., Xu, L., and Pan, X., Org. Biomol. Chem., 2018, vol. 16, p. 1816. https://doi.org/10.1039/c8ob00128f

16. Xu, L., Du, S., Li, Z., and Tian, Z., Heterocycles, 2018 , vol. 96 , no. 1, p. 74. https://doi.org/10.3987/com-17-13826

17. Liang, Y., Zhang, X., and MacMillan, D.W.C., Nature, 2018, vol. 559, p. 83. https://doi.org/10.1038/s41586-018-0234-8

18. Antilla, J.C., Baskin, J.M., Barder, T.E., and Buchwald, S.L., J. Org. Chem., 2004, vol. 69, no. 17, p. 5578. https://doi.org/10.1021/jo049658b

19. Gamonal, A., Brunet, E., Juanes, O., and RodriguezUbis, J.C., J. Photochem. Photobiol. (A), 2017, vol. 342, p. 53. https://doi.org/10.1016/j.jphotochem.2017.03.037

20. Reddy, A.V., Gogireddy, S., Dubey, P.K., Reddy, M.B., and Veeresh, B., J. Chem. Sci., 2015, vol. 127, p. 433. https://doi.org/10.1007/s12039-015-0792-3

21. Panova, V.A., Korsakov, M.K., Shetnev, A.A., and Filimonov, S.I., Mendeleev Commun., 2019, vol. 29, no. 1, p. 114. https://doi.org/10.1016/j.mencom.2019.01.039

22. Panova, V.A., Ivanovskii, S.A., Shetnev, A.A. Chirkova, Zh,V., Sudzilovskaya, T.N., and Filimonov, S.I., Russ. Chem. Bull., 2020, vol. 69, p. 1965. https://doi.org/10.1007/s11172-020-2986-1

23. Panova, V.A., Filimonov, S.I., Chirkova, Zh.V., Kabanova, M.V., Shetnev, A.A., Korsakov, M.K., Petzer, A., and Petzer, J.P., Bioorg. Chem., 2020, vol. 108, p. 104563. https://doi.org/10.1016/j.bioorg.2020.104563

24. Gierschner, J. and Park, S.Y., J. Mater. Chem. (C), 2013, vol. 1, p. 5818. https://doi.org/10.1039/C3TC31062K

25. Nishio, S., Higashiguchi, K., and Matsuda, K., Asian J. Org. Chem., 2014, vol. 3, p. 686. https://doi.org/10.1002/ajoc.201402024 